Research Article

\title{
Global Stability of a HIV-1 Model with General Nonlinear Incidence and Delays
}

\author{
Yaping Wang and Fuqin Sun \\ School of Science, Tianjin University of Technology and Education, Tianjin 300222, China \\ Correspondence should be addressed to Yaping Wang; ypwang1001@163.com and Fuqin Sun; sfqwell@163.com
}

Received 24 March 2013; Accepted 23 October 2013

Academic Editor: Vit Dolejsi

Copyright ( 2013 Y. Wang and F. Sun. This is an open access article distributed under the Creative Commons Attribution License, which permits unrestricted use, distribution, and reproduction in any medium, provided the original work is properly cited.

\begin{abstract}
A HIV-1 model with two distributed intracellular delays and general incidence function is studied. Conditions are given under which the system exhibits the threshold behavior: the disease-free equilibrium $E_{0}$ is globally asymptotically stable if $R_{0} \leq 1$; if $R_{0}>1$, then the unique endemic equilibrium $E_{1}$ is globally asymptotically stable. Finally, it is shown that the given conditions are satisfied by several common forms of the incidence functions.
\end{abstract}

\section{Introduction}

The global stability is analyzed for a general mathematical model of HIV-1 pathogenesis proposed by Nelson and Perelson [1]. The general model includes two distributed intracellular delays and a combination therapy with a reverse transcriptase inhibitor and a protease inhibitor. All incidence functions in those papers are the bilinear functions. However, there are some pieces of evidence showing that a bilinear infection rate might not be an effective assumption when the number of target cells is large enough (see [2-4] for the review of evidence and models). The aim of this paper is to establish global stability for a delay integrodifferential equation with a general incidence term $f(x, v)$, the conditions given here are similar to those given in [4] for the ODE case.

In this paper, we consider the following HIV-1 model with a side class of nonlinear incidence rates and distributed delays:

$$
\begin{aligned}
& \frac{d x(t)}{d t}=s-d_{T} x(t)-\left(1-n_{r t}\right) f(x(t), v(t)), \\
& \frac{d y(t)}{d t}=\left(1-n_{r t}\right) \int_{0}^{\infty} G_{1}(\xi) f(x(t-\xi),v(t-\xi)) d \xi-\delta y(t), \\
& \frac{d v(t)}{d t}=\left(1-n_{p}\right) N \delta \int_{0}^{\infty} G_{2}(\xi) y(t-\xi) d_{\xi}-c v(t),
\end{aligned}
$$

where $x, y$, and $v$ are the concentrations of uninfected target cells ( $\mathrm{T}$ cells), productively infected cells, and infectious virus, respectively.

The form of the incidence function $f$ is of fundamental importance. In this paper, we want to work with a function as general as possible but still possesses the properties necessary for conclusions to be made through mathematical analysis. Because of this, we will introduce conditions on $f$ with which it may appear technical. However, as shown in Section 5 , many commonly used incidence functions satisfy these conditions. For now, we assume only the following.

(A1) $f(x, v)$ is a nonnegative differentiable function on the nonnegative quadrant. Furthermore, $f(x, v)$ is positive if and only if both arguments are positive. The partial derivatives of $f$ on $x$ and $v$ are denoted by $f_{1}$ and $f_{2}$, respectively. In Sections 3 and 4 , it will be shown how the extra conditions on $f$ imply in the local and global stability of an endemic equilibrium.

For the purpose of convenience, we rewrite (1) as

$$
\begin{gathered}
\frac{d x(t)}{d t}=\lambda-m x(t)-\beta f(x(t), v(t)), \\
\frac{d y(t)}{d t}=\beta_{1} \int_{0}^{\infty} g_{1}(\xi) f(x(t-\xi), v(t-\xi)) d \xi-a y(t), \\
\frac{d v(t)}{d t}=k_{1} \int_{0}^{\infty} g_{2}(\xi) y(t-\xi) d \xi-\mu v(t),
\end{gathered}
$$


where $\lambda=s, m=d_{T}, \beta=1-n_{r t}, a=\delta, \mu=c, \beta_{1}=\left(1-n_{r t}\right) \alpha_{1}$, $k_{1}=\left(1-n_{p}\right) N \delta \alpha_{2}, g_{i}(\xi)=G_{i}(\xi) / \alpha_{i}, \alpha_{i}=\int_{0}^{\infty} G_{i}(\xi) d \xi, i=1,2$.

The paper is organized as follows. In Section 2, the basic reproduction number $R_{0}$ is determined and the equilibrium is found. The local stability of the equilibrium is studied in Section 3. The global dynamics are resolved in Section 4. In Section 5, examples are given of incidence functions which satisfy the assumptions that are used throughout the paper.

\section{Equilibria and $R_{0}$}

Assume the kernel functions $G_{1}$ and $G_{2}$ satisfy

$$
\begin{aligned}
& G_{i}(\xi) \geq 0, \quad \text { for } \xi \geq 0, \\
& 0<\int_{0}^{\infty} G_{i}(\xi) d \xi<1, \quad i=1,2 .
\end{aligned}
$$

For any given initial condition,

$$
\phi(\theta)=(x(\theta), y(\theta), v(\theta)) \in U C_{g}\left((-\infty, 0], \mathbf{R}_{+}^{3}\right),
$$

system (2) satisfies the hypotheses that are sufficient to ensure the existence, uniqueness, and continuity of solutions [5, Theorems 2.1-2.3]; for the notation of $U C_{g}$, see [6, page 46]. Recall that $\alpha_{i}=\int_{0}^{\infty} G_{i}(\xi) d \xi$ and $g_{i}(\xi)=G_{i}(\xi) / \alpha_{i}$; thus, $\int_{0}^{\infty} g_{i}(\xi) d \xi=1$. Then, the basic reproduction number $R_{0}$ defined in [7] can be rewritten as

$$
R_{0}=\frac{k_{1} \beta_{1}}{a \mu} f_{2}\left(E_{0}\right)
$$

and for any value of the parameters, the disease-free equilibrium of (2) is given by

$$
E_{0}=\left(x_{0}, y_{0}, v_{0}\right)=\left(\frac{\lambda}{m}, 0,0\right) .
$$

It is straightforward to show that if $R_{0} \leq 1$, then (2) has only one nonnegative equilibrium, which is called the infectionfree equilibrium $E_{0}$. The presence and number of endemic equilibrium depend on the form of the nonlinearity $f$, as well as the values of the parameters. In searching for equilibrium, we note that the equilibria of (2) are the same as the equilibria of the corresponding ordinary differential equation system. Here we give the following result.

Theorem 1. Let $(x(t), y(t), v(t))^{T}$ be the unique solution to system (2) with $x(0)>0, y(0)>0$, and $v(0)>0$. Then $x(t), y(t)$, and $v(t)$ are positive for all $t>0$. Moreover, the solution is bounded and thus exists globally.

Proof. Using the variation-of-constants formula, we obtain the positivity of $y(t)$ and $v(t)$.

For $x(t)$, we claim that if $x(0)>0$, then $x(t)>0$ for all $t>0$. Otherwise, there exists $t^{\prime}>0$ such that $x\left(t^{\prime}\right)=0$. Let $t_{0}$ be the first one that satisfies $x\left(t_{0}\right)=0$; then $t_{0}>0$ and

$$
\left.\frac{d x(t)}{d t}\right|_{t=t_{0}}=\lambda>0
$$

which means that $x(t)$ is increasing at $t=t_{0}$ and it is a contradiction.

Next we will show that every solution is also bounded. It follows from the first equation of (2) that $d x(t) / d t<\lambda-$ $m x(t)$. This implies that $\overline{\lim }_{t \rightarrow \infty} x(t) \leq \lambda / m$.

$$
\text { Let } V(t)=\left(\beta_{1} / \beta\right) \int_{0}^{\infty} g_{1}(\xi) x(t-\xi) d \xi+y(t) \text {; then }
$$

$$
\begin{aligned}
& \left.\frac{d V(t)}{d t}\right|_{(2)} \\
& =\frac{\beta_{1} \lambda}{\beta}-\frac{\beta_{1}(a-m)}{\beta} \int_{0}^{\infty} g_{1}(\xi) x(t-\xi) d \xi-a v(t) .
\end{aligned}
$$

Note that $\int_{0}^{\infty} g_{1}(\xi) x(t-\xi) d \xi$ is ultimately bounded; then there exist positive constants $C$ and $T_{0}$ such that

$$
\left.\frac{d V(t)}{d t}\right|_{(2)} \leq \frac{\beta_{1} \lambda}{\beta}+C-a V(t), \quad \forall t \geq T_{0} .
$$

This yields that $V(t)$ is eventually bounded and so is $y(t)$. By a similar argument, we can show the boundedness of $v(t)$. Therefore, the system (2) is point dissipative (see [1]) and hence the solution of (2) exists globally.

Theorem 2. If $R_{0}>1$, then there exists an endemic equilibrium $E_{1}=\left(x^{*}, y^{*}, v^{*}\right)$.

Proof. We look for solution $\left(x^{*}, y^{*}, v^{*}\right)$ of the equations $d x / d t=0, d y / d t=0$, and $d v / d t=0$. We first note that $(d x / d t)+\left(\beta / \beta_{1}\right)(d y / d t)=0$ implies $\lambda-m x^{*}-\beta f\left(x^{*}\right.$, $\left.v^{*}\right)+\left(\beta / \beta_{1}\right) a y^{*}=0$ and $k_{1} y^{*}-\mu v^{*}=0$, so $x^{*}=(\lambda-$ $\left.\left(a \beta / \beta_{1}\right) y^{*}\right) / m, v^{*}=k_{1} / \mu$. Let $H\left(y^{*}\right)=\beta_{1} f((\lambda-(a \beta /$ $\left.\left.\left.\beta_{1}\right) y^{*}\right) / m,\left(k_{1} / \mu\right) y^{*}\right)-a y^{*}$. Then, $d y(t) / d t=0$ whenever $H\left(y^{*}\right)=0$. Thus, any zero of $H$ in the interval $\left(0, \lambda \beta_{1} / a \beta\right)$ corresponds to an equilibrium with $x^{*}, y^{*}, v^{*}>0$, that is, an endemic equilibrium.

Since $f(0, v)=f(x, 0)=0$, it follows that $H(0)=0$ and $H\left(\lambda \beta_{1} / a \beta\right)<0$. The function $H$ is continuous and so a sufficient condition for $H$ to have a zero in $\left(0, \lambda \beta_{1} / a \beta\right)$ is that $H$ is increasing at 0 . Thus, there is an endemic equilibrium if

$$
0<\frac{d H}{d y}(0)=\beta_{1}\left(-\frac{a \beta}{m \beta_{1}}\right) f_{1}\left(E_{0}\right)+\frac{k_{1} \beta_{1}}{\mu} f_{2}\left(E_{0}\right)-a .
$$

Since $f(x, 0)=0$ for all $x$, it follows that $f_{1}\left(E_{0}\right)=0$ and so (10) is equivalent to $R_{0}=\left(k_{1} \beta_{1} / a \mu\right) f_{2}\left(E_{0}\right)>1$.

\section{Local Stability of the Equilibria}

Theorem 3. If $R_{0}<1$, then $E_{0}$ is locally asymptotically stable.

The proof is normal, so we omitted it. We now give conditions on $f$ that are used here and after to show the locally and globally asymptotically stability of the endemic equilibrium. As a precondition, we assume that $R_{0}>1$ to guarantee the existence of endemic equilibrium $E_{1}=\left(x^{*}, y^{*}, v^{*}\right)$. Consider the following.

(A2) $\operatorname{sgn}\left(f\left(x, v^{*}\right)-f\left(x^{*}, v^{*}\right)\right)=\operatorname{sgn}\left(x-x^{*}\right)$ for all $x \geq 0$. 
(A3) For all $x, v>0, f(x, v) / f\left(x, v^{*}\right)$ is in the closed interval with endpoints at 1 and $v / v^{*}$.

(A4) Either $f_{1}\left(x^{*}, v^{*}\right)>0$ or $f_{2}\left(x^{*}, v^{*}\right)<f\left(x^{*}, v^{*}\right) / v^{*}$.

Theorem 4. If $R_{0}>1$, and (A2), (A3), and (A4) hold, then any endemic equilibrium which exists is locally asymptotically stable.

Proof. The characteristic equation of the linearization of (2) at endemic equilibrium $E_{1}=\left(x^{*}, y^{*}, v^{*}\right)$ is

$$
\begin{aligned}
0= & \left(m+\lambda+\beta f_{1}\left(E_{1}\right)\right)(a+\lambda)(\mu+\lambda) \\
& -(m+\lambda) \beta_{1} k_{1} f_{2}\left(E_{1}\right) \\
& \times \int_{0}^{\infty} g_{1}(\xi) e^{-\lambda \xi} d \xi \int_{0}^{\infty} g_{2}(\xi) e^{-\lambda \xi} d \xi .
\end{aligned}
$$

We demonstrate that all zeros of it have negative real part. Since (A2) and (A3) hold, we get that $0 \leq \beta f_{1}\left(E_{1}\right)$ and $0 \leq$ $f_{2}\left(E_{1}\right) \leq a \mu / \beta_{1} k_{1}$. Suppose that $\lambda$ has nonnegative real part, we deduce

$$
\begin{aligned}
& \left|(m+\lambda) \beta_{1} k_{1} f_{2}\left(E_{1}\right) \int_{0}^{\infty} g_{1}(\xi) e^{-\lambda \xi} d \xi \int_{0}^{\infty} g_{2}(\xi) e^{-\lambda \xi} d \xi\right| \\
& \quad \leq|m+\lambda| \beta_{1} k_{1} f_{2}\left(E_{1}\right) \\
& \quad \leq\left|\left(m+\lambda+\beta f_{1}\left(E_{1}\right)\right)(a+\lambda)(\mu+\lambda)\right|,
\end{aligned}
$$

and so the solutions $\lambda$ with nonnegative real part if and only if all of the inequalities in (12) are in fact equal. The final inequality is strict unless $f_{1}\left(E_{1}\right)=0$ (and $\lambda=0$ ). The second last inequality is strict unless $f_{2}\left(E_{1}\right)=f\left(E_{1}\right) / v^{*}$. Assumption (A4) implies at least one is strict. Thus, the endemic equilibrium $E_{1}$ is locally asymptotically stable.

\section{Global Stability of the Equilibria}

Theorem 5. If $R_{0} \leq 1$, then $E_{0}$ is globally stable; that is,

$$
\lim _{t \rightarrow \infty}(x(t), y(t), v(t))=E_{0}
$$

Proof. Let $K(t)=K_{1}(t)+K_{2}(t)+K_{3}(t)$ with

$$
\begin{aligned}
& K_{1}(t)=\frac{1}{2}\left(x(t)-\frac{\lambda}{m}\right)^{2}+\frac{\lambda \beta}{m \beta_{1}} y(t)+\frac{a \lambda \beta}{k_{1} m \beta_{1}} v(t), \\
& K_{2}(t)=\frac{\lambda \beta}{m} \int_{0}^{\infty} H_{1}(\xi)(f(x(t-\xi), v(t-\xi)) \\
& \left.\quad+f_{2}\left(x_{0}, 0\right) v(t-\xi)\right) d \xi, \\
& K_{3}(t)=\frac{a \lambda \beta}{m \beta_{1}} \int_{0}^{\infty} H_{2}(\xi) y(t-\xi) d \xi,
\end{aligned}
$$

where $H_{i}(t)=\int_{t}^{\infty} g_{i}(\xi) d \xi, i=1,2$.
It is clear that $K(t) \geq 0$ and $K(t)=0$ if and only if $x(t)=$ $\lambda / m, y(t)=v(t)=0$. The derivative of $K_{1}$ along the solution of (2) is

$$
\begin{aligned}
\left.\frac{d K_{1}(t)}{d t}\right|_{(2)}= & \left(x(t)-\frac{\lambda}{m}\right)(\lambda-m x(t)-\beta f(x(t), v(t))) \\
& -\frac{a \lambda \beta}{m \beta_{1}} y(t)-\frac{a \lambda \beta \mu}{k_{1} m \beta_{1}} v(t) \\
& +\frac{\lambda \beta}{m} \int_{0}^{\infty} g_{1}(\xi) f(x(t-\xi), v(t-\xi)) d \xi \\
& +\frac{a \lambda \beta}{m \beta_{1}} \int_{0}^{\infty} g_{2}(\xi) y(t-\xi) d \xi
\end{aligned}
$$

Noticing that $H_{1}(0)=1, H_{1}(\infty)=0$, and $d H_{1}(t)=-g_{1}(t) d t$, and using integration by parts, we calculate the derivatives of $K_{2}$ and $K_{3}$

$$
\begin{aligned}
\left.\frac{d K_{2}(t)}{d t}\right|_{(2)}= & \frac{\lambda \beta}{m} f(x, v)+\frac{\lambda \beta}{m} f_{2}\left(x_{0}, 0\right) \\
& -\frac{\lambda \beta}{m} \int_{0}^{\infty} g_{1}(\xi) f(x(t-\xi), v(t-\xi)) d \xi \\
& -\frac{\lambda \beta}{m} f_{2}\left(x_{0}, 0\right) \int_{0}^{\infty} g_{1}(\xi) v(t-\xi) d \xi . \\
\left.\frac{d K_{3}(t)}{d t}\right|_{(2)}= & \frac{a \lambda \beta}{m \beta_{1}}\left(y(t)-\int_{0}^{\infty} g_{2}(\xi) y(t-\xi) d \xi\right) .
\end{aligned}
$$

Thus,

$$
\begin{aligned}
\left.\frac{d K(t)}{d t}\right|_{(2)}= & -m\left(x(t)-\frac{\lambda}{m}\right)^{2} \\
& -\beta x(t) f(x(t), v(t))-\frac{a \lambda \beta \mu}{m \beta_{1} k_{1}}\left(1-R_{0}\right) v(t) \\
& -\frac{\lambda \beta}{m} f_{2}\left(x_{0}, 0\right) \int_{0}^{\infty} g_{1}(\xi) v(t-\xi) d \xi \leq 0 .
\end{aligned}
$$

Recalling that $f$ is nonnegative and is positive if and only if both arguments are positive, we must have that $\left\{E_{0}\right\}$ is the largest invariant subset of $\{d K / d t=0\}$; then the global stability of $E_{0}$ follows from the classical Lyapunov-LaSalle invariance principle (see [6], Theorem 2.7.1).

Theorem 6. If $R_{0}>1$, and (A2) and (A3) hold, then $E_{1}$ is globally stable in $D=R_{+}^{3} \backslash E_{0}$.

Proof. Let $W(t)=W_{1}(x(t), y(t), v(t))+W_{2}(x(t), y(t), v(t))+$ $W_{3}(x(t), y(t), v(t))$. 
With

$$
\begin{aligned}
& W_{1}(x(t), y(t), v(t))=\int_{0}^{\infty} g_{1}(\xi) U(t, \xi) d \xi \\
& W_{2}(x(t), y(t), v(t)) \\
& \quad=\frac{\beta}{\beta_{1}} y^{*} h\left(\frac{y}{y_{*}}\right)+\beta f\left(x^{*}, v^{*}\right) \int_{0}^{\infty} H_{1}(\xi) h\left(\frac{v(t-\xi)}{v_{*}}\right) d \xi,
\end{aligned}
$$$$
W_{3}(x(t), y(t), v(t))
$$$$
=\frac{a \beta}{k_{1} \beta_{1}} F\left(v(t), v^{*}\right)+\frac{a \beta}{\beta_{1}} \int_{0}^{\infty} H_{2}(\xi) F\left(y(t-\xi), y^{*}\right) d \xi,
$$

where $U(t, \xi)=x(t-\xi)-x^{*}-\int_{x^{*}}^{x(t-\xi)}\left(f\left(x^{*}, v^{*}\right) / f\left(\sigma, v^{*}\right)\right) d \sigma$, $h(t)=t-1-\ln t$, and $F\left(u, u^{*}\right)=u-u^{*} \ln u-\left(u^{*}-u^{*} \ln u^{*}\right)$; it is easy to see that $h(t)$ has the strict global minimum $h(1)=$ 0 for $t>0, F\left(u, u^{*}\right) \geq F\left(u^{*}, u^{*}\right)=0$ for $u>0$ and any positive constant $u^{*}$. By Theorem 1 , all solutions are positive and bounded. Thus, $W(t)$ is well defined and $W(t) \geq 0$, in which the equality holds if and only if $y(t)=y^{*}, v(t)=v^{*}$ and $x(t-\xi)=x^{*}, y(t-\xi)=y^{*}, v(t-\xi)=v^{*}$ for almost all $\xi \in[0, \infty)$. For clarity, the derivatives of $W_{1}, W_{2}$, and $W_{3}$ will be calculated separately and then combined to obtain $d W / d t$.

First, $d W_{1} / d t=\int_{0}^{\infty} g_{1}(\xi)(d U(t, \xi) / d t) d \xi$, where

$$
\begin{aligned}
\frac{d U(t, \xi)}{d t}= & \left(1-\frac{f\left(x^{*}, v^{*}\right)}{f\left(x(t-\xi), v^{*}\right)}\right) \\
& \times(\lambda-m x(t-\xi)-\beta f(x(t-\xi), v(t-\xi))) \\
= & -m\left(x(t-\xi)-x^{*}\right)\left(1-\frac{f\left(x^{*}, v^{*}\right)}{f\left(x(t-\xi), v^{*}\right)}\right) \\
& +\beta f\left(x^{*}, v^{*}\right)\left(1-\frac{f(x(t-\xi), v(t-\xi))}{f\left(x^{*}, v^{*}\right)}\right. \\
& -\frac{f\left(x^{*}, v^{*}\right)}{f\left(x(t-\xi), v^{*}\right)} \\
& \left.+\frac{f(x(t-\xi), v(t-\xi))}{f\left(x(t-\xi), v^{*}\right)}\right) .
\end{aligned}
$$

Since $\lambda=m x^{*}+\beta f\left(x^{*}, v^{*}\right)$. That is,

$$
\begin{aligned}
\frac{d W_{1}}{d t}= & -m \int_{0}^{\infty} g_{1}(\xi)\left(x(t-\xi)-x^{*}\right) \\
& \times\left(1-\frac{f\left(x^{*}, v^{*}\right)}{f\left(x(t-\xi), v^{*}\right)}\right) d \xi \\
& +\beta f\left(x^{*}, v^{*}\right) \\
& \times \int_{0}^{\infty} g_{1}(\xi)\left(1-\frac{f(x(t-\xi), v(t-\xi))}{f\left(x^{*}, v^{*}\right)}\right.
\end{aligned}
$$

$$
\begin{gathered}
\left.-\frac{f\left(x^{*}, v^{*}\right)}{f\left(x(t-\xi), v^{*}\right)}\right) d \xi \\
+\beta f\left(x^{*}, v^{*}\right) \int_{0}^{\infty} g_{1}(\xi) \frac{f(x(t-\xi), v(t-\xi))}{f\left(x(t-\xi), v^{*}\right)} d \xi .
\end{gathered}
$$

Next, we calculate $d W_{2} / d t$. Consider

$$
\begin{aligned}
& \frac{d W_{2}}{d t}=\beta f\left(x^{*}, v^{*}\right) \\
& \times \int_{0}^{\infty} g_{1}(\xi) \frac{f(x(t-\xi), v(t-\xi))}{f\left(x^{*}, v^{*}\right)} \\
& \times\left(1-\frac{y^{*}}{y}\right) d \xi-\frac{\beta a}{\beta_{1}} y(t)+\frac{\beta}{\beta_{1}} a y^{*} \\
& +\beta f\left(x^{*}, v^{*}\right) \int_{0}^{\infty} g_{1}(\xi) \\
& \times\left(h\left(\frac{v}{v^{*}}\right)-h\left(\frac{v(t-\xi)}{v^{*}}\right)\right) d \xi \\
& =\beta f\left(x^{*}, v^{*}\right) \int_{0}^{\infty} g_{1}(\xi) \\
& \times\left[\frac{f(x(t-\xi), v(t-\xi))}{f\left(x^{*}, v^{*}\right)}\right. \\
& \left.-\frac{y^{*}}{y} \frac{f(x(t-\xi), v(t-\xi))}{f\left(x^{*}, v^{*}\right)}-\frac{y^{*}}{y}\right] d \xi \\
& +\beta f\left(x^{*}, v^{*}\right) \int_{0}^{\infty} g_{1}(\xi) \\
& \times\left[1+h\left(\frac{v}{v^{*}}\right)-h\left(\frac{v(t-\xi)}{v^{*}}\right)\right] d \xi
\end{aligned}
$$

Using the fact that $H_{2}(0)=1, H_{2}(\infty)=0$, and $d H_{2}=$ $-g_{2}(t) d t$, we get

$$
\begin{aligned}
\frac{d W_{3}}{d t}= & \frac{a \beta}{k_{1} \beta_{1}}\left(1-\frac{v^{*}}{v}\right)\left(k_{1} \int_{0}^{\infty} g_{2}(\xi) y(t-\xi) d \xi-\mu v(t)\right) \\
& -\frac{a \beta}{\beta_{1}} \int_{0}^{\infty} H_{2}(\xi) \frac{d F\left(y(t-\xi), y^{*}\right)}{d \xi} d \xi \\
= & -\frac{a \beta}{\beta_{1}} \int_{0}^{\infty} g_{2}(\xi) \frac{v^{*}}{v} y(t-\xi) d \xi-\frac{a \beta \mu}{k_{1} \beta_{1}}\left(v-v^{*}\right) \\
& +\frac{a \beta}{\beta_{1}} y(t)+\frac{a \beta}{\beta_{1}} y^{*} \int_{0}^{\infty} g_{2}(\xi) \ln \frac{y(t-\xi)}{y} d \xi \\
= & -\beta f\left(x^{*}, v^{*}\right) \int_{0}^{\infty} g_{2}(\xi) h\left(\frac{v^{*}}{v} \frac{y(t-\xi)}{y}\right) d \xi \\
& +\beta f\left(x^{*}, v^{*}\right) \int_{0}^{\infty} g_{1}(\xi)\left(h\left(\frac{y}{y^{*}}\right)-h\left(\frac{v}{v^{*}}\right)\right) d \xi .
\end{aligned}
$$


The third quality holds since $y^{*}=\left(\beta_{1} / a\right) f\left(x^{*}, v^{*}\right), v^{*}=$ $\left(k_{1} / \mu\right) y^{*}$. We conclude that

$$
\begin{aligned}
\frac{d W}{d t}=-m \int_{0}^{\infty} & g_{1}(\xi)\left(x(t-\xi)-x^{*}\right) \\
& \times\left(1-\frac{f\left(x^{*}, v^{*}\right)}{f\left(x(t-\xi), v^{*}\right)}\right) d \xi \\
& -\beta f\left(x^{*}, v^{*}\right) \int_{0}^{\infty} g_{2}(\xi) h\left(\frac{v^{*}}{v} \frac{y(t-\xi)}{y}\right) d \xi \\
& +\beta f\left(x^{*}, v^{*}\right) \int_{0}^{\infty} g_{1}(\xi) C(\xi) d \xi,
\end{aligned}
$$

where

$$
\begin{aligned}
C(\xi)=2 & -\frac{f\left(x^{*}, v^{*}\right)}{f\left(x(t-\xi), v^{*}\right)}+\frac{f(x(t-\xi), v(t-\xi))}{f\left(x(t-\xi), v^{*}\right)} \\
& -\frac{y^{*}}{y} \frac{f(x(t-\xi), v(t-\xi))}{f\left(x^{*}, v^{*}\right)}-\frac{y}{y^{*}} \\
& +h\left(\frac{y}{y^{*}}\right)-h\left(\frac{v(t-\xi)}{v^{*}}\right) \\
= & -h\left(\frac{f\left(x^{*}, v^{*}\right)}{f\left(x(t-\xi), v^{*}\right)}\right) \\
& -h\left(\frac{y^{*}}{y(x(t-\xi), v(t-\xi))} \frac{f\left(x^{*}, v^{*}\right)}{y}\right) \\
& +h\left(\frac{f(x(t-\xi), v(t-\xi))}{f\left(x(t-\xi), v^{*}\right)}\right)-h\left(\frac{v(t-\xi)}{v^{*}}\right) .
\end{aligned}
$$

Since the function $h(t)$ is monotone on each side of 1 and is minimized at $t=1$, (A3) implies that $h((f(x(t-\xi), v(t-$ $\left.\xi))) /\left(f\left(x(t-\xi), v^{*}\right)\right)\right) \leq h\left(v(t-\xi) / v^{*}\right)$. Therefor, noting that $h \geq 0$ and (A2), we have $d W / d t \leq 0$. So the solutions must tend to $M$, the largest invariant subset of $\{d W / d t=0\}$.

To have $d W / d t$ equal zero, it is necessary to have ( $x(t-$ $\left.\xi)-x^{*}\right)\left(1-\left(f\left(x^{*}, v^{*}\right) / f\left(x(t-\xi), v^{*}\right)\right)\right)=0$ for almost every $\xi \in[0, \infty)$, which holds if and only if $x(t)=x^{*} ; v^{*} y(t-\xi)=$ $v(t) y(t)$ and $y^{*} f(x(t-\xi), v(t-\xi))=y(t) f\left(x^{*}, v^{*}\right)$ for almost every $\xi \in[0, \infty)$.

Since $M$ is invariant with respect to (2), we get

$$
\begin{aligned}
\frac{d y(t)}{d t} & =\beta_{1} \int_{0}^{\infty} g_{1}(\xi) f(x(t-\xi), v(t-\xi)) d \xi-a y(t) \\
& =\beta_{1} \int_{0}^{\infty} g_{1}(\xi) \frac{y(t) f\left(x^{*}, v^{*}\right)}{y^{*}} d \xi-a y(t)=0 .
\end{aligned}
$$

So this determines $y(t)$ to be a constant. Using $v^{*} y(t-\xi)=$ $v(t) y(t)$, we obtain $v(t)=v^{*}$ and this yields that $y(t)=y^{*}$. Thus, each element of $M$ satisfies $x(t)=x^{*}, y(t)=y^{*}$, and $v(t)=v^{*}$ for all $t>0$. This shows that

$$
\lim _{t \rightarrow \infty}(x(t), y(t), v(t))=\left(x^{*}, y^{*}, v^{*}\right)=E_{1} .
$$

This completes the proof.

\section{Examples}

We now give some examples of incidence functions for which the hypotheses are satisfied.

Example 1 (mass action). Let $f(x, v)=x v$. The hypotheses (A1)-(A4) are satisfied and so global dynamics are determined by the magnitude of $R_{0}$. The global behavior of this model was previously studied in $[1,8]$ and was fully resolved in [9].

Example 2 (saturating incidence). Let $f(x, v)=\beta x(t) v(t) /$ $(1+\alpha x(t))$ for some constant $\beta>0, \alpha>0$. The hypotheses (A1)-(A4) are satisfied and so global dynamics are determined by the magnitude of $R_{0}$. The global properties of this model were studied in [10].

Example 3 (Holling type II incidence). Let $f(x, v)=$ $\beta x(t) v(t) /(1+v(t))$ for some constant $\beta>0$. The hypotheses (A1)-(A4) are satisfied and so global dynamics are determined by the magnitude of $R_{0}$. The global properties of this model were studied in [11].

Example 4 (Beddington-DeAngelis incidence). Let $f(x, v)=$ $x(t) v(t) /(1+a x(t)+b v(t))$ for some constants $a>0$ and $b>0$. The hypotheses (A1)-(A4) are satisfied and so global dynamics are determined by the magnitude of $R_{0}$. The global properties were studied in $[12,13]$.

\section{Acknowledgment}

This work was supported by the Technology Development Foundation of Higher Education of Tianjin (20081003).

\section{References}

[1] P. W. Nelson and A. S. Perelson, "Mathematical analysis of delay differential equation models of HIV-1 infection," Mathematical Biosciences, vol. 179, no. 1, pp. 73-94, 2002.

[2] A. Korobeinikov, "Stability of ecosystem: global properties of a general predator-prey model," Mathematical Medicine and Biology, vol. 26, no. 4, pp. 309-321, 2009.

[3] A. Korobeinikov, "Global asymptotic properties of virus dynamics models with dose dependent parasite reproduction and virulence, and nonlinear incidence rate," Mathematical Medicine and Biology, vol. 26, pp. 225-239, 2009.

[4] A. Korobeinikov, "Global properties of infectious disease models with nonlinear incidence," Bulletin of Mathematical Biology, vol. 69, no. 6, pp. 1871-1886, 2007.

[5] J. K. Hale and J. Kato, "Phase space for retarded equations with infinite delay," Funkcialaj Ekvacioj, vol. 21, no. 1, pp. 11-41, 1978.

[6] Y. Kuang, Delay Differential Equations with Applications in Population Dynamics, vol. 191 of Mathematics in Science and Engineering, Academic Press, Boston, Mass, USA, 1993.

[7] O. Diekmann, J. A. P. Heesterbeek, and J. A. J. Metz, "On the definition and the computation of the basic reproduction ratio $R_{0}$ in models for infectious diseases in heterogeneous populations," Journal of Mathematical Biology, vol. 28, no. 4, pp. 365382, 1990. 
[8] H. Zhu and X. Zou, "Impact of delays in cell infection and virus production on HIV-1 dynamics," Mathematical Medicine and Biology, vol. 25, pp. 99-112, 2009.

[9] S. Q. Liu and L. Wang, "Global stability of an HIV-1 model with distributed intracellular delays and a combination therapy," Mathematical Biosciences and Engineering, vol. 7, no. 3, pp. 675$685,2010$.

[10] G. Huang, H. Yokoi, Y. Takeuchi, T. Kajiwara, and T. Sasaki, "Impact of intracellular delay, immune activation delay and nonlinear incidence on viral dynamics," Japan Journal of Industrial and Applied Mathematics, vol. 28, no. 3, pp. 383-411, 2011.

[11] D. Li and W. Ma, "Asymptotic properties of a HIV-1 infection model with time delay," Journal of Mathematical Analysis and Applications, vol. 335, no. 1, pp. 683-691, 2007.

[12] G. Huang, W. B. Ma, and Y. Takeuchi, "Global properties for virus dynamics model with Beddington-DeAngelis functional response," Applied Mathematics Letters, vol. 22, no. 11, pp. 16901693, 2009.

[13] B. Y. Fu and W. B. Ma, "Stability analysis of an HIV-1 infection model with Beddington-DeAngelis functional response," Mathematics in Practice and Theory, vol. 39, pp. 71-79, 2009 (Chinese). 


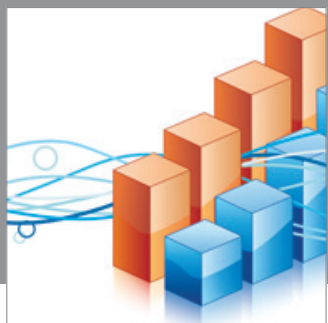

Advances in

Operations Research

mansans

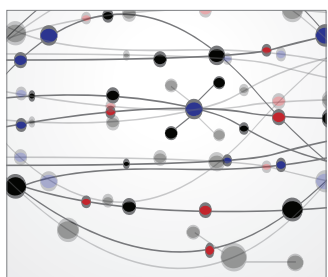

The Scientific World Journal
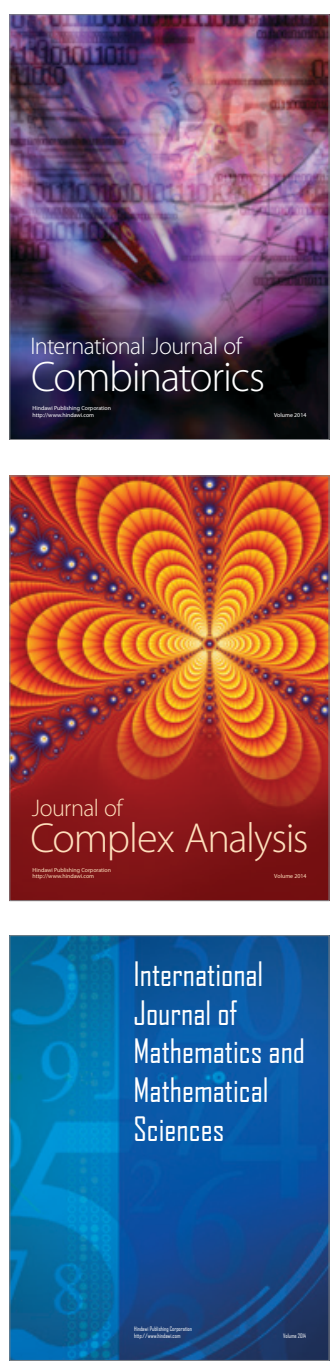
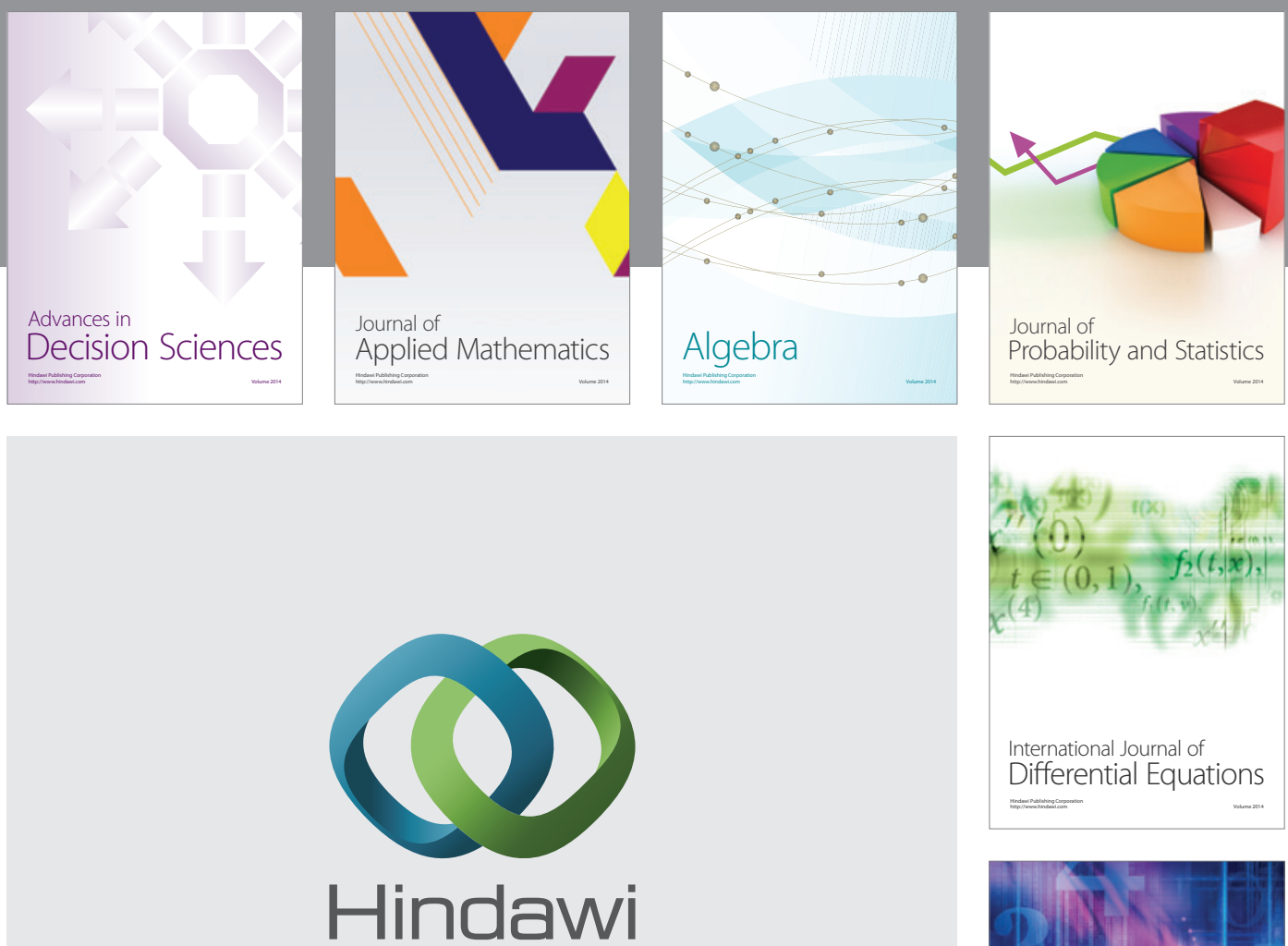

Submit your manuscripts at http://www.hindawi.com
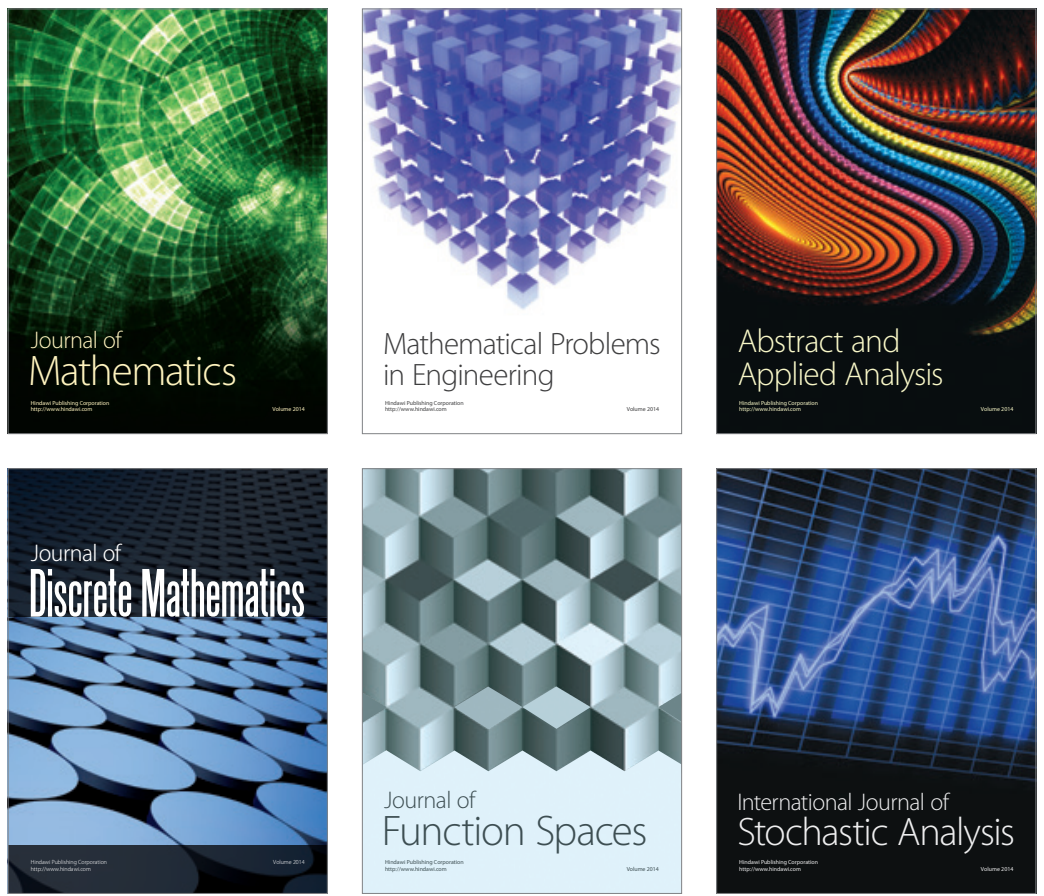

Journal of

Function Spaces

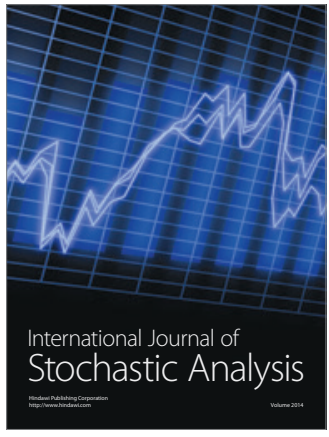

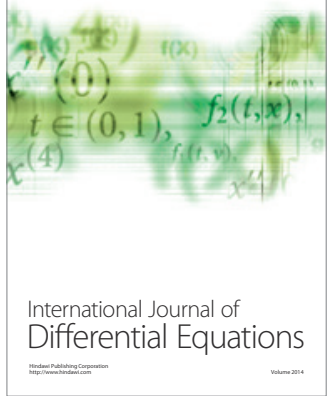
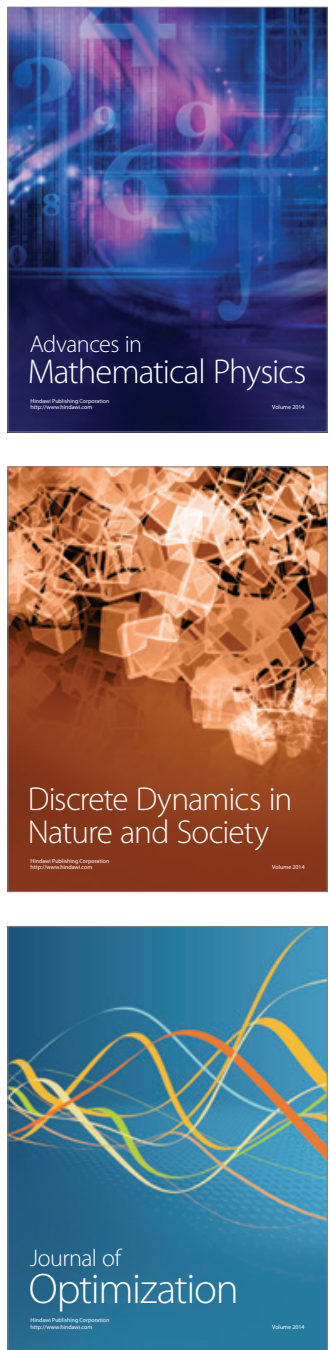